\title{
The monitoring of an existing cast iron tunnel with distributed fibre optic sensing (DFOS)
}

\author{
C. Y. Gue ${ }^{1}$ M. Wilcock ${ }^{2}$ M. M. Alhaddad ${ }^{2}$ - M. Z. E. B. Elshafie ${ }^{2}$. \\ K. $\operatorname{Soga}^{2} \cdot$ R. J. Mair ${ }^{2}$
}

Received: 14 January 2015/Revised: 19 March 2015/Accepted: 23 March 2015/Published online: 29 March 2015

(C) The Author(s) 2015. This article is published with open access at Springerlink.com

\begin{abstract}
Instrumentation is vital to tunnelling projects for validation of design assumptions, monitoring of trigger levels during construction and also serving as a feedback loop to understand the deformation mechanisms of the particular problem which could be used to improve future designs. It can also be used for long term monitoring of the tunnel for maintenance purposes. Distributed fibre optic sensing (DFOS) systems based on brillouin optical time domain reflectometry are able to provide continuous and distributed strain measurements to be taken along the entire length of, for example, an existing cast iron tunnel where the fibre optic cable length is fully attached. This enables engineers to understand the stresses and strains that develop within the lining caused by external influences; which in this case, the construction of a new tunnel directly underneath it, rather than relying on discrete point measurements of displacement from conventional methods of monitoring. Nonetheless, proper installation of DFOS is of paramount importance to obtain high quality data. This paper aims to provide some practical guidance on the planning and installation of DFOS and presents a brief case study on the monitoring of London's Royal Mail tunnel during the construction of the large Crossrail platform tunnel, located directly below it at Liverpool Street Station. It demonstrates the potential benefits of using such systems in complex tunnelling scenarios.
\end{abstract}

C. Y. Gue

cyg20@cam.ac.uk

1 Schofield Centre, University of Cambridge, Cambridge CB3 OEL, UK

2 Department of Engineering, University of Cambridge, Cambridge CB2 1PZ, UK
Keywords Tunnels - Effects of tunnelling on existing tunnels · Distributed fibre optic strain sensing · Field monitoring

\section{Introduction}

In urban environments where a complicated underground tunnel network exists, it is inevitable for new tunnels to be constructed in close proximity to existing underground structures. These existing structures, depending on their constitutive materials and construction methods could be very susceptible to tunnelling induced ground movements. As pointed out by Peck [12], the success of tunnel construction extends beyond the ability of the new tunnel lining to resist forces that it will be subjected to throughout its lifetime. It is also necessary to control and minimise any adverse effects on adjacent existing structures.

Over the last few decades, advancement in analytical models and computational numerical methods [3, 10, 14] has allowed engineers to analyse and design tunnel linings with a high degree of confidence by incorporating moderately conservative soil and structural parameters. As far as construction methods are concerned, the development of the earth pressure balance and slurry shield tunnel boring machines (TBMs) have allowed machine-driven tunnels to be constructed safely in complex ground conditions. Tunnels constructed with sprayed concrete linings (SCL) in soft ground are becoming more common as well in competent soil. Construction of new tunnels, albeit more complex, is less problematic as technology has developed. Nonetheless, the effects of tunnelling on adjacent structures remain challenging as they are not well understood; in practice, with surging demand for transport infrastructure in urban environments, tunnelling close below existing 
tunnels is becoming increasingly common and thus progressively more important.

The limited knowledge on the response of existing tunnels to new tunnel construction warrants higher conservatism for the design of mitigation and prevention measures. Mitigation and remedial works could be very costly and time consuming should the movements of the existing tunnel exceed the typically very stringent trigger levels specified in practice. Therefore, the key is to obtain feedback through monitoring to improve the understanding and predictions on the response of the existing tunnel due to tunnelling. This can only be achieved through the use of appropriate instrumentation to measure the important parameters at relevant locations within the existing tunnel, employing proper installation methods.

Automated total stations (ATS) are often used for tunnel monitoring and it provides displacement measurements at the target points. On the other hand, distributed fibre optic strain sensing is relatively new in the construction industry with many of its sub-variations still under research and development. The measured strain profile can potentially provide information that the conventional displacement-based monitoring systems cannot give. Based on the collective experience of the authors and of the researchers at the Centre for Smart Infrastructure and Construction (CSIC) at Cambridge University, some practical installation aspects of DFOS for the monitoring of an existing cast iron tunnel will be discussed in this paper. Results will be presented and discussed to show the superior practical information such systems can offer to asset owners, designers and construction professionals. Appropriate installation procedures are key for DFOS to achieve a successful outcome.

\section{Installation considerations}

\subsection{Parameters of measurement}

\subsubsection{Site background}

Currently the largest tunnel construction project in Europe, Crossrail aims to increase the capacity of London's rail system by $10 \%$, linking Reading and Heathrow in the west of London and Shenfield and Abbey Woods in the east by 2018. It consists of $118 \mathrm{~km}$ of rail, of which $42 \mathrm{~km}$ are twin underground tunnels in central London, serving 40 stations. There are eight new sub-surface stations being constructed in central London.

At Liverpool Street Station, a new $11 \mathrm{~m}$ diameter platform tunnel has been constructed directly beneath the Royal Mail tunnel, as shown in Fig. 1, in a parallel alignment by means of the open-face sprayed concrete lining (SCL) tunnelling method; the tunnelling is principally in London Clay, a very stiff competent soil. The clear separation between the invert of the Royal Mail tunnel and the crown of the new Crossrail tunnel decreases from around $2-1 \mathrm{~m}$ at the installation location, as shown in Fig. 1.

The construction of the Royal Mail tunnel was completed in 1917 to convey mail from eastern to western sorting offices in London. Constructed wholly in London Clay at an average depth of $27 \mathrm{~m}$ below ground level, the Royal Mail tunnel is made of cast iron rings, each consisting of seven grey cast iron segments, approximately $0.5 \mathrm{~m}$ wide, forming a $2.97 \mathrm{~m}$ external diameter tunnel. It stretches for $10.5 \mathrm{~km}$, with eight stations. In 2003 due to high running costs, operations were halted in the Royal Mail tunnel and the tunnel still remains closed. Since the Royal Mail tunnel is disused, it presented an ideal trial site for various state-of-the-art monitoring systems to be tested.

\subsubsection{Mode of deformation}

One of the main uncertainties design engineers face when encountering such a scenario is the mode of deformation of the segmental cast iron linings in the longitudinal direction; as the tunnel experiences tunnelling induced ground movement beneath it. The bolted rings could either respond as an intact and continuous unit where the bending mode deformation would be dominant or the segmental rings could behave discontinuously such that each ring displaces or shears vertically in relation to each other in the shearing mode deformation [1] as shown in Fig. 2. Induced stresses in the bolts and linings will differ significantly depending on the deformation mode.

In cross-section, as the new tunnel is constructed, the existing cast iron tunnel linings will ovalise as soil move downwards to the newly constructed cavity due to stress relief (see Fig. 11). It is important to note here that the existing linings will have a certain stress regime in place from its original construction and thus, depending on the mode of tunnelling induced stresses, the cast iron could potentially become overstressed, leading to its ultimate limit state. A conventional approach to this problem is to adopt an analytical solution such as that of Duddeck and Erdmann [3] to estimate the maximum allowable deformation of the tunnel ring and set trigger levels in terms of the percentage of ring convergence.

While displacement at a prescribed point can easily be tracked by means of ATS, the main aim from such an approach is to quantify the amount of stresses that are induced in the lining in terms of absolute displacement at any point in the tunnel. With very limited targets along the internal tunnel circumference, much interpolation and assumptions are required to back analyse and estimate the 

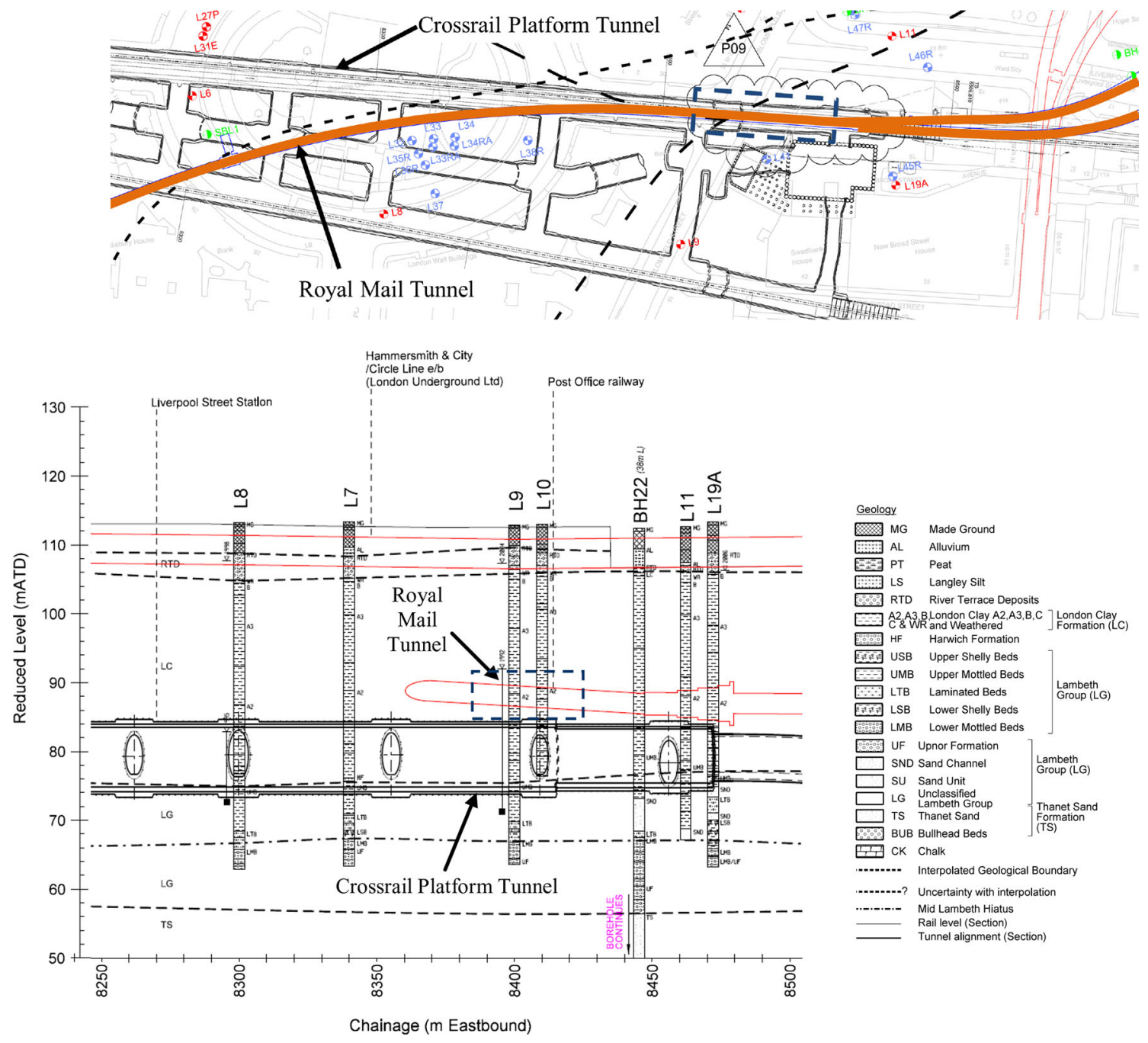

Fig. 1 (Top) alignment of Royal Mail tunnel and Crossrail platform tunnel at London Liverpool Street Station. (Bottom) longitudinal section view of Royal Mail and Crossrail platform tunnel with soil profile

stresses from the displacements of each target. Nonetheless, the ATS method is usually adopted because of its wide use and simplicity to measure displacements. Conventionally, measurement of displacement is easier but inferring the resultant strains and stresses from displacements could be very challenging.

In short, the Royal Mail tunnel required an instrumentation system which could measure cross sectional strains due to ovalisation as well as longitudinal strains, both of which were induced by the construction of Crossrail's platform tunnel. With this in mind, several state-of-the-art instrumentation systems such as DFOS, wireless displacement and tilt sensors and photogrammetry were deployed [1]. This paper will only focus on the DFOS system although some monitoring results from the other instrumentation systems will be included for comparison.

\subsection{Type of DFOS system}

Many variations of fibre optic strain sensing systems exist, some of which have been commercialised and some are still in the stage of research and development. This paper will only discuss briefly the two most commonly used distributed fibre optic systems: Brillouin Optical Time Domain Reflectometry (BOTDR) and Brillouin Optical Time Domain Analyses (BOTDA). A general overview description of both systems will be given below.

\subsubsection{Brillouin optical time domain reflectometry (BOTDR)}

The BOTDR analyser supplies an incident light signal through the single mode fibre optic cables via a pump laser where most of the signal will pass through the cable but a 


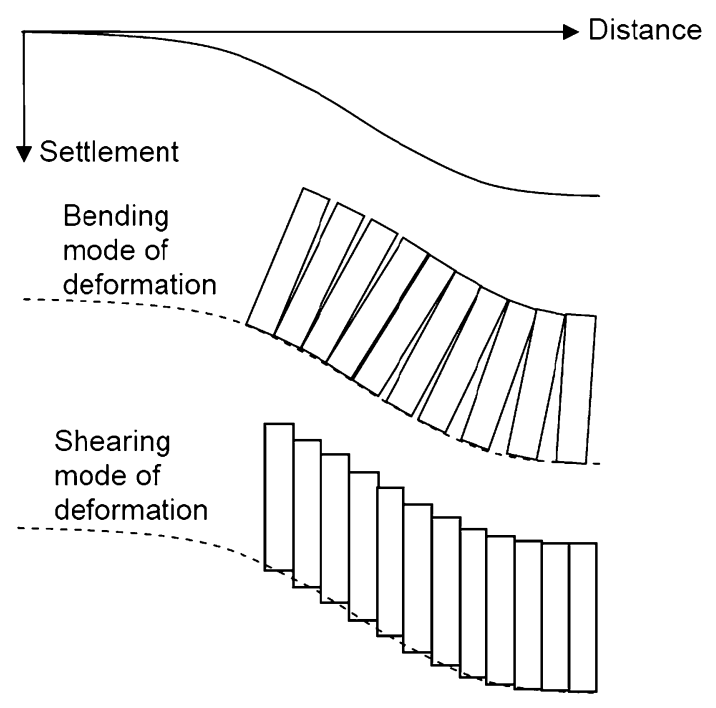

Fig. 2 Bending and shear deformation modes for the existing cast iron lining tunnel in the longitudinal direction. (After Alhaddad et al. [1])

small portion of it will be reflected back into the analyser as backscattered light. Within the Brillouin scattering process, the backscattered light experiences a frequency shift, which is linearly proportional to strain. The BOTDR analyser is able to measure and record the frequency shift, and hence strain, at every point in the cable. Depending on the time for the signal to return to the analyser, the distance along the cable in which the strain occurred can be determined, effectively transforming the entire fibre optic cable to a continuous strain sensor itself [7-9].

Since it relies on the return signal from a single light source, only single access to either end of the cable is required. However, its dependence on the backscattered light means that it is more susceptible to the effects of tight bends during installation (macrobends) and minute pinch or squeezing of the fibre optic core (microbends) which could result in a significant power loss such that the analyser may not be able to register the return signal if it is too weak.

Among other factors, the range of strain within the fibre optic cable, the cable length, and the average count of readings and spatial resolution will all affect the data acquisition time significantly.

\subsubsection{Brillouin optical time domain analyses (BOTDA)}

The BOTDA works through stimulated Brillouin scattering. Similar to BOTDR, a pulse signal is generated at one end of the cable but at the same time, a continuous wave signal is emitted from the other end. Thus, this method requires a closed measurement loop from the analyser to the sensing cable and back to the analyser again. This stimulation creates significantly higher signal to noise ratio, enabling low powered pump lasers to be used [4]. Similar to BOTDR, the return signal experiences a linearly dependent strain induced frequency shift, which is captured by the analyser.

Due to the nature of the system where it receives signals from only half of the loop, an extension cable of a length at least equal to the length of the sensor cable attached to the structure for strain measurement is required. In comparison to BOTDR, the data acquisition time for BOTDA is much faster for the same instrumented system at generally higher accuracies.

\subsubsection{Discussion}

Despite the advantages of BOTDA, the main drawback is the inherent requirement for a complete measurement loop. Fibre optic cables are very fragile in nature and breakages are common in the harsh and unpredictable construction site environment. Should a breakage occur, the BOTDA system will not be able to take any readings at all unless the breakage is repaired. On the other hand, for BOTDR, strain measurements can be taken up to the point of breakage. In the event of a single breakage, the instrumented section can be salvaged by taking readings from both ends of the cable before combining it in the analysis.

The BOTDR system was selected for reliability in this particular case study for Royal Mail tunnel; the Yokogawa AQ8603 analyser was employed for the instrumentation. It can achieve an accuracy of $\pm 30 \mu \varepsilon$, which was deemed sufficient for the identification of the deformation mechanism. For the cases where the site environment can be well and reliably controlled, the BOTDA system would be the preferred option.

\subsection{Survivability of fibre optic cable}

Fibre optic cables are very fragile due to the brittleness of the core, which measures only $125 \mu \mathrm{m}$ in diameter for single mode cables. To increase its durability, these cores are generally coated with thick jackets for added protection. The cost of the cable per meter run increases with the degree of protection it provides. Justifying the cost of the cables would depend on the risk assessment for the environment in which the fibre optic cables would be installed. For example, fibre optic cables cast in concrete would need a higher degree of protection as opposed to those that would be glued on the surface of existing structures.

In addition to the added cost, a more durable cable translates to a stiffer cable. Depending on the choice of attachment, prestressing the cables (inducing a pre-tension to allow capturing of compressive strains) will generate large forces at anchoring points, which could increase the 

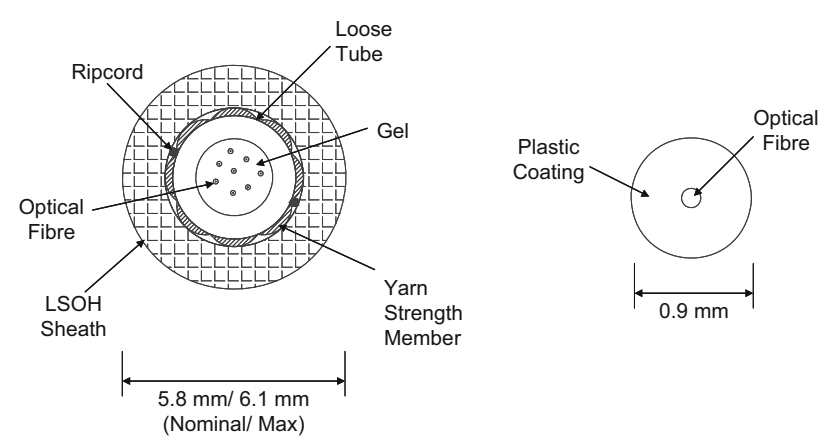

Fig. 3 (Left) excel 8C 9/125 loose tube LSOH cable for temperature sensing. (Right) hitachi single mode single core tight buffered fibre optic cable for strain sensing

risk of creep. Furthermore, having multiple protective layers and coatings increases the complexity of working with the cable on site, i.e. stripping cables for splicing. The balance between durability, practicality and cost needs to be managed with care.

For the case study discussed in this paper, the fibre optic cables were attached to the linings of an existing tunnel. Figure 3 shows the two types of cables that were used in the field instrumentation. The risk of damage to the cable was deemed to be sufficiently low such that a simple Hitachi single mode single core tight-buffered cable (Fig. 3 right) was a viable choice as the strain sensing cable. This cable was previously calibrated for elastic strain deformation of up to $6000 \mu \varepsilon$ [7]. Excel 8C 9/125 loose tube cables (Fig. 3 left) served as an extension cable that had the capability of temperature sensing due to its gel filled core which prevents mechanical strain transfer from the jacket.

\subsection{Methods of fibre optic attachment}

\subsubsection{Continuous gluing}

To exploit the continuous strain sensing capabilities of DFOS, it is always recommended to bond the entire length of the cable onto the existing structure. Cross-sectional fibre optic cables in this case study were attached using this continuous gluing method, as shown in Fig. 4.

Cleaning of the cast iron surface is vital to achieve good bond between the cable and the lining. Gentle tapping proved to be the most efficient method to remove surface grout revealing the actual cast iron surface. Remaining dirt and grease were then cleaned by means of soap and water.

Low pull magnets are useful to seat and temporarily hold the fibre optic cable in place along the circumference of the flanges; across the radial joints for the application and setting of epoxy. For this reason, a light and flexible cable, such as the Hitachi single mode single core tight buffered cable, which could be seated to a curved surface proved to be advantageous.

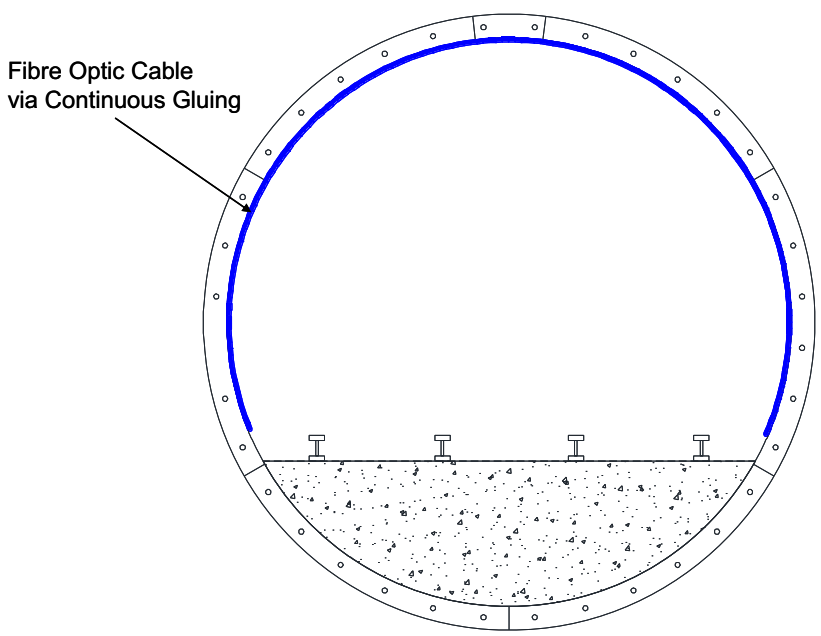

Fig. 4 Cross sectional fibre optic cable attachment via continuous gluing with Araldite 2021 epoxy

Fully bonded fibre optic cable sections are confined by the movement of the structure. As a result, measurements of both tensile and compressive strains are possible without the need for prestraining. The curvature along the circumference of the tunnel lining would in fact induced small tensile strains that aid identification of each cross section during data analyses. The low initial strains on the cables allow it to accommodate for the slightly larger movements across the segmental joints.

Each cross-sectional cable was connected to each other on the same continuous cable where the connecting cable runs freely along the sides of the tunnel lining, resting on existing cables and conduits (see Fig. 7). This provided a zero mechanical strain reference, which could be used as a counter check for temperature compensation in the analysis stage (this is discussed in "Sect. 2.5").

However, unwanted movements of the connecting cable could occur during installation (i.e. the fibre optic cable was dragged along during the repositioning of power cables for tools and lighting during installation). As an improvement over the free running cables which was used in this field instrumentation, undesired straining of the 'zero strain' cable can be prevented by containing a loose loop of fibre optic cables with a standard length (i.e., $5 \mathrm{~m}$ ) inside a closed box for minimal disturbance. It must be highlighted that the ingress and egress of the cable should not be fixed or held in place to prevent breakage in the event that the cable is dragged accidentally.

While unintentional cable movements may result in a shorter zero strain length, the benefit of keeping the cable intact with this flexibility outweighs the slight inconsistency in length. With the exception that the cable is dragged for a considerable distance, the zero-strain loop would be identifiable once the change in strains is computed in the analysis. In the worst case scenario where a cross sectional 
cable is damaged during installation or monitoring, zerostrain loops between the affected regions could be used as an emergency length of cable, eliminating the need to transport replacement cables to the monitored section within the tunnel.

\subsubsection{Spot gluing}

Spot gluing means that the fibre optic cable is only fixed at a series of predetermined points. In contrast with the continuous gluing method, strain measurements from spot gluing will only be representative for the lengths between the fixity points. Nonetheless, there are situations which render the continuous gluing method less practical. Direct line of sight along the intrados surface at the tunnel crown was not possible with the flanges and existing electrical conduits in place. Continuous gluing would have required the fibre optic cable to be bent at four locations per ring. This greatly increases the risk of cable breakage and could inflict significant power loss from macro bends.

In this field study, a series of $\mathrm{L}$ brackets measuring $110 \mathrm{~mm} \quad$ (height) $\times 50 \mathrm{~mm} \quad$ (width) $\times 80 \mathrm{~mm}$ (length) $\times 10 \mathrm{~mm}$ thick were bolted in the mid-section of each pan to create a raised platform which cleared all obstacles and maintained a straight line of sight. The fibre optic cable was then prestrained before gluing it on the $\mathrm{L}$ bracket with Araldite 2021 epoxy.

Figure 5 shows how fixity or anchorage was provided by Fujikura IC-Rock clamps that were pre-attached to a L-bracket; which is shorter to compensate for the dimension of the clamp, to maintain a constant level for the fibre optic cables. The procedure of prestraining for an installation span between the first and the Nth ring (anchorage point) is shown in Fig. 5.

As the tunnel deforms longitudinally in the bending mode, the gap between consecutive rings expands or contracts depending on their location with respect to the hogging and sagging zones, causing a change in strain in

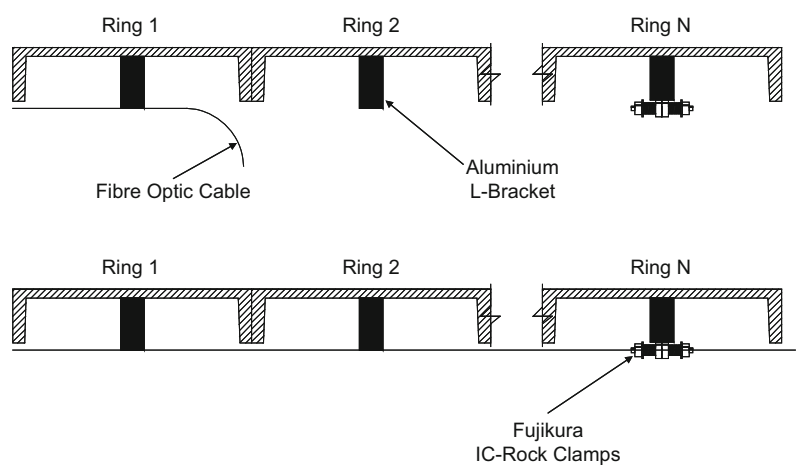

Fig. 5 Procedure of prestraining fibre optic cables along the crown of existing tunnel the fibre optic cable that can be measured. In this scenario, prestrain was necessary to measure compressive strains as a reduction in tensile strains.

Insufficient initial tensile prestrain would cause the fibre optic cable to sag under compressive stress. Thus it is important to estimate the maximum compressive strain that the system would experience and apply a suitable margin of safety for the required prestrain. In the other extreme, excessive prestrain will lead to non-elastic behaviour of the fibre optic cable which ultimately leads to cable breakage. Based on the authors' experience, a prestrain value of between 1000 and $3000 \mu \varepsilon$ is sufficient for most cases but this should be exercised with caution as the requirement and performance of different structures may vary.

The exact value of prestrain is not important to be known since the relative change in strain is of primary interest. However, large discrepancies of prestrain values between two consecutive lengths will result in erratic results or spikes over the transition region of the two prestrain lengths. It is strongly recommended that prestraining is carried out by controlled means such as with a newton spring balance.

A fibre optic cable with a relatively high axial stiffness requires a larger force to achieve the set prestrain value and this force will be transferred to the anchoring points, increasing the effect of creep. Disturbing forces on the L-brackets were minimised by the use of Hitachi single mode single core tight-buffered cable, which has a low stiffness of approximately $10 \mathrm{~N}$ per $1 \%$ strain.

As the installation span increases, the amount of sag of the fibre optic cable due to its self-weight increases accordingly. Working with a very extensible (i.e. low axial stiffness) cable meant that large amount of strains could have been induced just to keep the cable taut. This can easily exceed the range of the required prestrain. To reduce this risk, installation spans should be kept as small as practical to minimise sagging. An installation span of $8 \mathrm{~m}$ was initially adopted in this field instrumentation.

A separate catenary calculation with the known cable unit weight can be carried out to justify installation spans based on the maximum allowable cable sag. Naturally, risk of large cable sag is higher for cables that are more durable since the added protection effectively increases its unit weight.

It is important to note that the consideration of allowable cable sag should only be used as a rough guide because the relative position of linings in the actual tunnel may not be perfectly levelled and aligned vertically. Due to this reason, along with the constraints of permitted working hours, a final average installation span of $13 \mathrm{~m}$ was achieved. This exercise is useful only as an indication of the installation span. The use of a spring balance for prestraining works would allow better control and consistency. 


\subsection{Temperature compensation}

Strain measured by the analyser is a combination of mechanical and thermal strain. It is important to separate one from the other and this can be done through the presence of a separate or part of the same fibre optic cable, which is unattached to the structure, running alongside the strain sensing cables; where they are subjected to the same temperature variations.

Excel 8C 9/125 loose tube fibre optic cables in this field installation served dual purpose; as an economical extension cable as well as for temperature compensation. The cable consists of 8 fibre optic cores suspended in a gel filled core. This effectively breaks the mechanical bond between the outer jacket and the fibre optic cores, thus only thermal strains are measured by the cores. This can then be used to offset the relative strains measured for a separate strain cable during the analysis.

The thermal strain component, $\Delta \varepsilon_{\text {Thermal }}$ can be separated into two parts as shown in Eqs. 1.0 and 1.1. Temperature induced apparent strain, $\alpha_{\mathrm{a}}$ is a constant of $19.47 \times 10^{-6}$ for a standard single mode fibre at $1550 \mathrm{~nm}$ wavelength, and $\alpha_{\mathrm{n}}$ is the net thermal expansion for the jacket, which can be significantly higher than that of the core itself [7].

$\Delta \varepsilon_{\text {Thermal }}=\left(\alpha_{\mathrm{a}}+\alpha_{\mathrm{n}}\right) \Delta T$

$\alpha_{\text {Thermal }}=\alpha_{\mathrm{a}}+\alpha_{\mathrm{n}}$

Type and make of commercially available fibre optic cables vary considerably in the market and will have different $\alpha_{n}$ values depending on the material of the jacket. Hence a direct compensation for thermal strain across different cables cannot be carried out without proper conversion to account for the variation in $\alpha_{\text {Thermal }}$. It should be noted here that when fibre optic cables are cast in concrete, the thermal expansion of the strain cables is governed by that of concrete.

In the case where temperature and strain cables have different known $\alpha_{\mathrm{n}}$ and $\alpha_{\text {Thermal }}$ values, the equivalent thermal strain of the strain cable can be converted from the temperature cable via the relationship shown in Fig. 6 where subscript $T$ and $S$ refer to temperature and strain cable, respectively.

Once the equivalent thermal strain on the strain cable is computed, a direct subtraction can be made from the measured strains from the strain cable to obtain the true mechanical strains (Eq. 2.0).

$\Delta \varepsilon_{\text {Mechanical }}=\Delta \varepsilon_{\text {Measured }-S}-\Delta \varepsilon_{\text {Thermal }-S}$

Level of noise in the measured data is variable and its magnitude will influence the method of temperature compensation. The first method, as mentioned above, is the direct subtraction of equivalent thermal strain on the strain
$\Delta T$

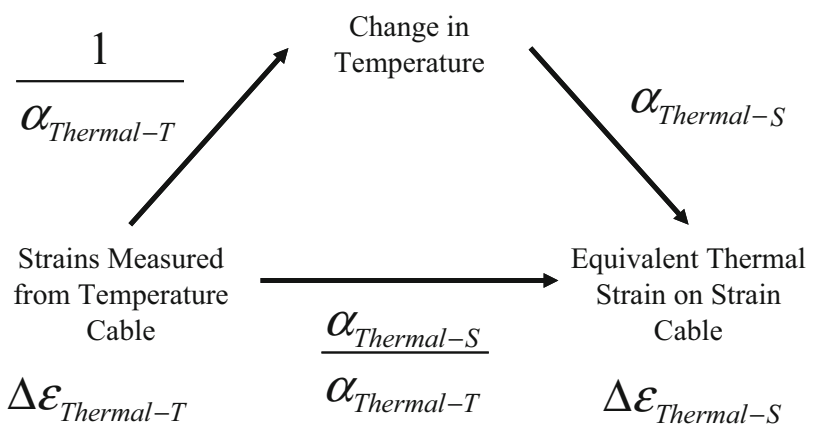

Fig. 6 Diagram showing the conversion from thermal strains measured from temperature cable to an equivalent thermal strain on the strain cable

cable. In an ideal situation where the noise level is very low, this may prove to be the easiest method. However, if the thermal strains are noisy, correcting for them may generate additional noise for the final computed mechanical strain.

In the latter case, provided that the particular length of cable of interest is assumed to have uniform temperature variation over time, one might choose to subtract the strain data from an averaged equivalent thermal strain.

Alternatively, if a zero strain loop is available, mechanical strain can be computed by correcting the strain measurements directly in order maintain a nil strain over the zero strain length. Once again, this method only applies if the temperature variation is uniform throughout.

\subsection{Optical budget loss estimation}

Each fibre optic analyser system has a threshold on the minimum signal power that it can read which is termed the optical budget loss. Power signal is described in terms of decibels. This governs the amount of power loss that is acceptable for the system.

Power loss in the system can occur through four areas: cable attenuation, loss through splice, loss through connectors and installation factors. Impurities in the fibre optic cable create attenuation within the cable itself, thus a longer cable system suffers larger power loss in a similar manner to voltage drop across a long electrical cable. Since it is an intrinsic attenuation, the power loss along the cable is unavoidable but this is usually small in comparison to other factors. Manufacturers of the fibre optic cables will be able provide details of the cable attenuation. Alternatively, calibration can be done beforehand.

Imperfections in the splice connection will induce power loss. Competency of the operator is crucial to ensure these losses, albeit quantifiable, are kept to a minimum. Thus, the idea is to minimise the number of splices and connectors in 
the system. Arc fusion splice which has a better performance than its mechanical counterpart is highly recommended within practical constraints.

Finally, installation effects such as macro and microbends, including other unforeseen circumstances in the installation or extension cable, will add on to the budget loss but these are much more difficult to estimate and are less predictable. The rule of thumb for telecommunication fibre optic installation is to allocate $3 \mathrm{~dB}$ loss margin for this factor. Depending on site conditions, analyser requirements and experience, this rule of thumb may change.

The sum of these losses, shown in Eq. 3.0, gives an indication of the adequacy of the system during the planning stages. Modifications such as reducing or omitting mechanical splices altogether may be an option should the optical budget loss exceed the specification of the analyser.

$\mathrm{OBL}=n_{\text {con }} L_{\text {con }}+n_{\text {splice }} L_{\text {splice }}+a_{\text {cable }} L_{\text {cable }}+M$

where OBL is the optical budget loss, $n_{\text {con }}$ is the number of connectors, $L_{\mathrm{con}}$ is the estimated power loss per connector, $n_{\text {splice }}$ is the number of splices, $L_{\text {splice }}$ is the estimated power loss per splice, $a_{\text {cable }}$ is the estimated attenuation of cable per $1 \mathrm{~km}, L_{\text {cable }}$ is the length of fibre optic cable in $\mathrm{km}$ and $M$ is the margin for unforeseen circumstances. For example, the conservative budget loss estimation for this installation with two connectors, each with a loss of $0.25 \mathrm{~dB}$ were connected to a $0.56 \mathrm{~km}$ long fibre optic cable with an attenuation of $0.5 \mathrm{~dB} / \mathrm{km}$, along with a total of four fusion splices that were carried out; two for connectors and another two for repairs with a loss of $0.1 \mathrm{~dB}$ each, would result in the final optical budget loss of $4.18 \mathrm{~dB}$, inclusive of the margin of $3 \mathrm{~dB}$.

\section{Results and analyses}

\subsection{Overall layout of fibre optic instrumentation}

The fibre optic instrumentation layout in the Royal Mail tunnel is shown in Fig. 7. It consists of a $40 \mathrm{~m}$ long longitudinal section with five cross sections at $10 \mathrm{~m}$ intervals. The fibre optic cable is extended via Excel 8C 9/125 Loose Tube cables to the top of an access shaft. All splices were kept in an enclosed splice holder for protection. Direction of tunnelling for the new Crossrail platform tunnels within the monitored section are from ring R2950 in decreasing order to ring R2870.

\subsection{Cross sectional behaviour}

Monitoring was carried out over a period of 11 months from 16th of April 2013 to 14th of March 2014. A pilot tunnel of approximately $6 \mathrm{~m}$ in diameter was constructed

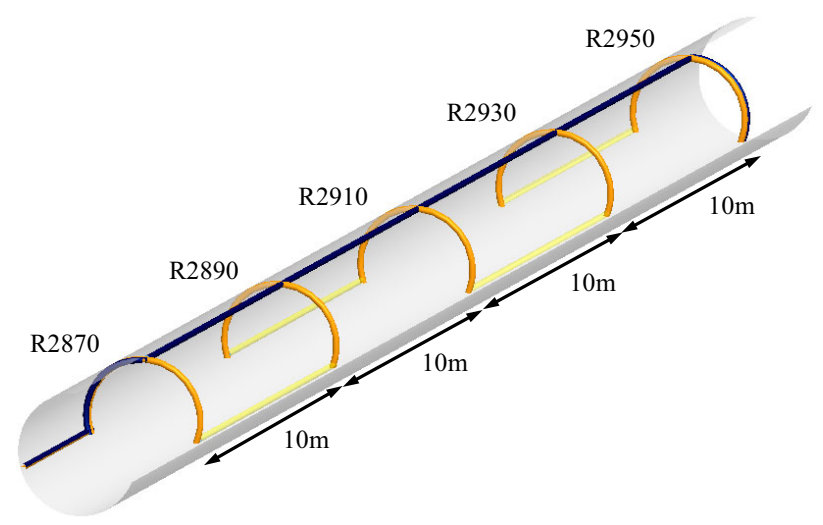

Fig. 7 Fibre optic cable instrumentation layout at Royal Mail tunnel

prior to the full enlargement to approximately $11 \mathrm{~m} \mathrm{di}$ ameter. All of the tunnelling works were carried out via open faced tunnelling with sprayed concrete lining.

A typical ground profile with the position of the existing Royal Mail tunnel and the new Crossrail platform tunnel are shown in Fig. 8. The crown of the final enlarged platform tunnel will sit at an average distance of $2 \mathrm{~m}$ below the invert of the Royal Mail tunnel.

As the monitoring stretched over a long period of time, for clarity, only data from three stages are presented and discussed in detail, namely the initial construction stage, pilot tunnel construction and the tunnel enlargement. Figure 9 shows the cumulative change in strains for ring R2950 at three construction stages where the location is shown in Fig. 10. The horizontal axis on Fig. 9 corresponds to the length or position of the cable along the attached circumference of the cast iron lining; the vertical axis reports the change in strains (expressed as a percentage).

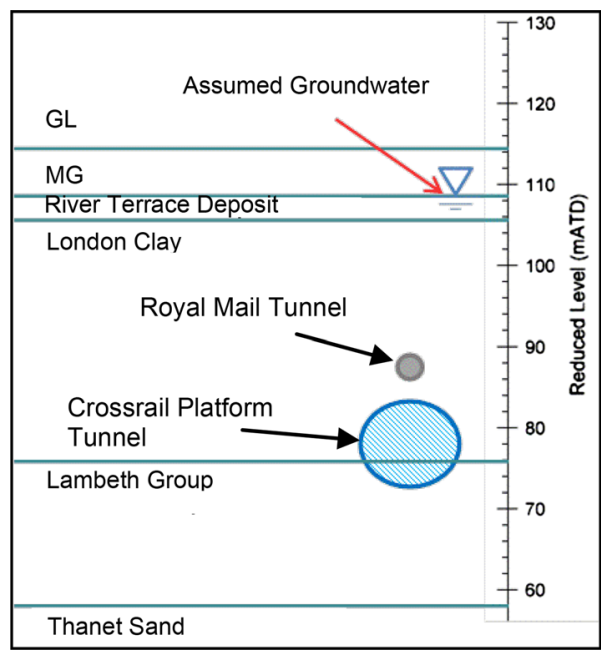

Fig. 8 Typical ground profile with position of the Royal Mail Tunnel and Crossrail Platform Tunnel 


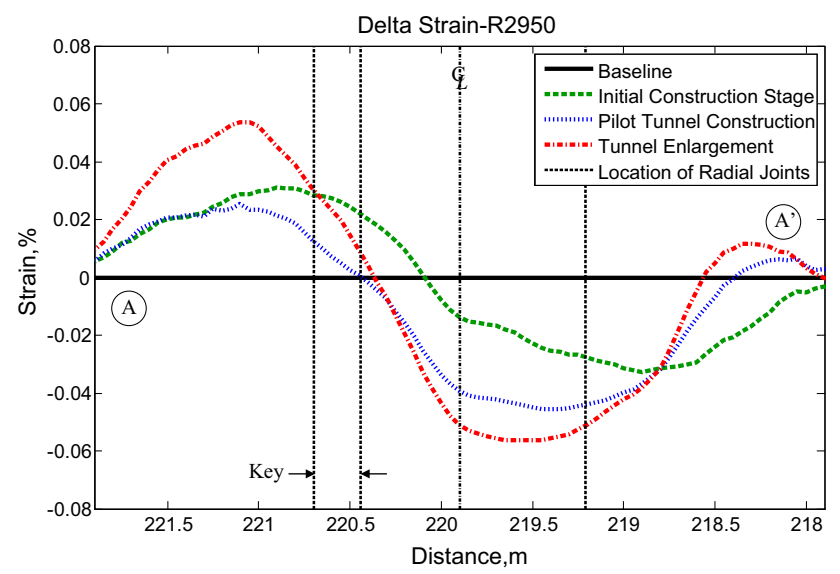

Fig. 9 Plot of cumulative change in strain for ring R2950 at various construction stages

Strain increments were computed from the baseline data collected on 16th of April 2013, denoted by the solid horizontal line in Fig. 9. There was no significant construction within the vicinity of the monitored section until late July 2013 when an access passage was constructed between the east and westbound platform tunnels, which will be referred as the initial construction stage. This access was located at an offset to the southwest direction of ring R2950 of the existing Royal Mail Tunnel as shown in Fig. 10.

As ground movements are caused by construction of the access tunnel, the existing Royal Mail tunnel deforms in a skewed ellipse directed towards the new access tunnel. Therefore at ring R2950 of the Royal Mail tunnel, denoted by $\mathrm{A}-\mathrm{A}^{\prime}$, the lining closest to the access tunnel (south wall) experienced tensile strains while the far side (north wall) went into compression as shown in Fig. 9. The deformation pattern can be visualised qualitatively based on the strain patterns shown in Fig. 11.

Pilot tunnel construction approached the monitored section by mid-September 2013, which caused the ring to ovalise vertically as the tunnelling works progressed underneath ring R2950. At this stage the trend of strains take on the dominant profile where the south wall was in tension followed by compression at the crown before returning to tension in the north wall.

The pattern of strain profiles remained unchanged for the tunnel enlargement in January 2014 since the direction of tunnelling was constant but with much larger strain values that were in-line with the construction of a much larger $11 \mathrm{~m}$ diameter tunnel at smaller clear distances below the Royal Mail tunnel. Computing the equivalent stress. Similar trends were consistently recorded on all other instrumented rings with the exception of R2870 where the cable was damaged during installation.

The maximum recorded cumulative strains on the cross section for both tension and compression were in the region of $550 \mu \varepsilon(0.055 \% \varepsilon)$. Computing the equivalent stress in the cast iron lining would bring it to around $66 \mathrm{MPa}$. It is important to point out that the fibre optic cables were glued continuously along the circumference and across the joints between adjacent cast iron segments. The presence of wood packing in the joints would result in a zone of low stiffness which is averaged out by the analyser over its spatial resolution of $1 \mathrm{~m}$. Therefore, the values measured were a composite between the strains over the joints and those sustained by the cast iron panels.

A deconvolution process would need to be carried out to quantify the actual amount of strains that developed in the

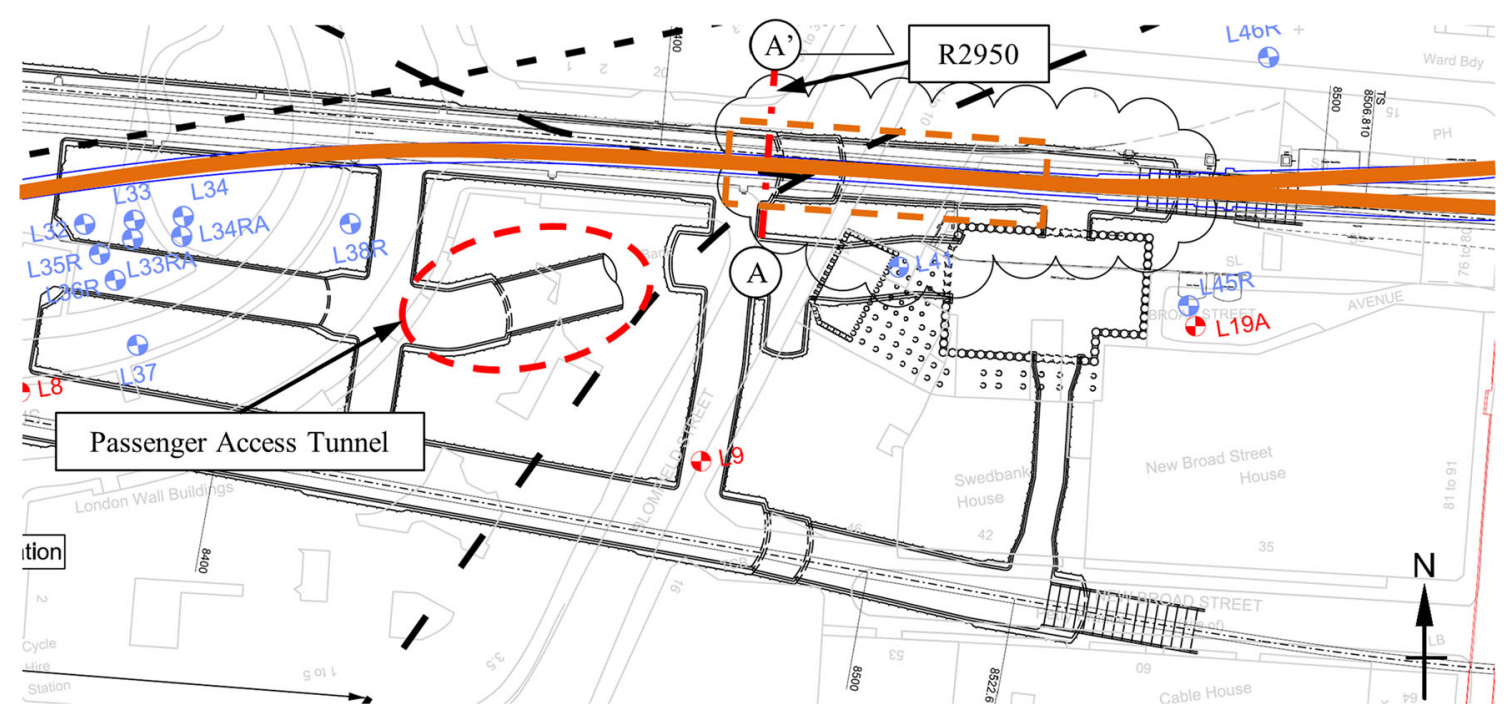

Fig. 10 Location of passenger access tunnel in relation to ring R2950 


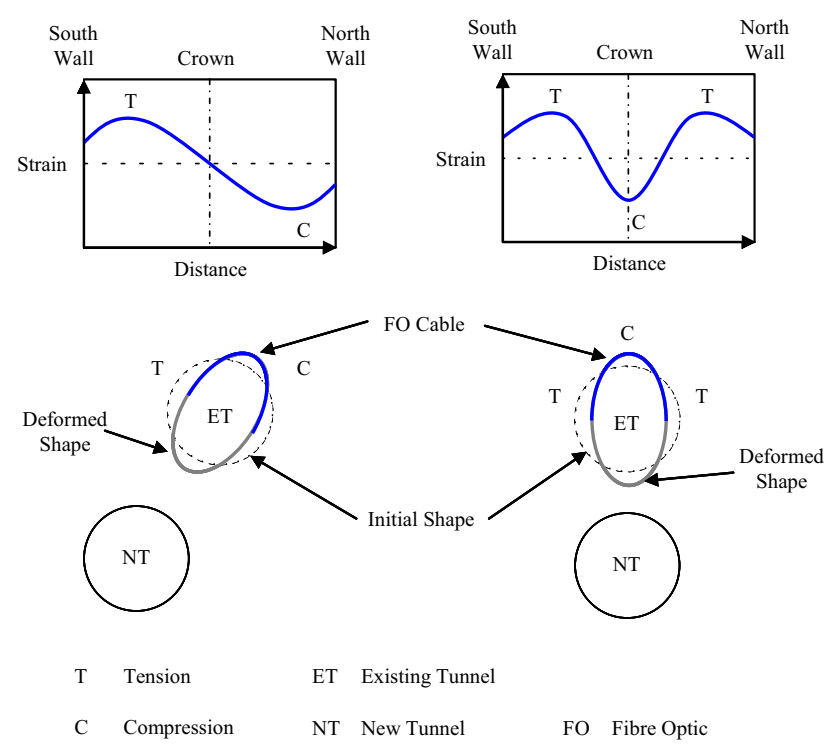

Fig. 11 Visualisation of tunnel deformation from fibre optic strain data

cast iron segments themselves. This process would require the strains at each joint to be known. Unfortunately, local strain gauges were not installed over the joints in this field instrumentation; hence the maximum actual stresses within the cast iron segments would in fact be lower than the directly computed value of $66 \mathrm{MPa}$. Nonetheless, the measured strains would still be representative of the behaviour of the cast iron ring as a whole.

\subsection{Longitudinal behaviour}

Based on empirical evidences, tunnelling induced longitudinal Greenfield surface ground movements can be represented by a cumulative normal distribution curve $[2,11]$ as detailed in Fig. 12.

It is expected that a similar "bow wave" movement would be transferred to the Royal Mail tunnel. Crown settlement of a typical static point measured by ATS is shown in the top graph of Fig. 13. The horizontal axis represents time of construction in days while the vertical axis denotes the crown ATS target settlements in $\mathrm{mm}$. The vertical dashed lines at day 44 and 187 marks the time where the static point enters the influence zone of the pilot and full tunnel enlargement construction stages, respectively. Large changes in settlements could be seen over the next few days at these two points in time as the tunnel construction progressed until it exited the influence zone. There were no construction activities between days 76 and 186 therefore the fibre optic analyser was mobilised to another site during this period.

The lower graph of Fig. 13 presents the strains recorded from the longitudinal fibre optic cable at the same location

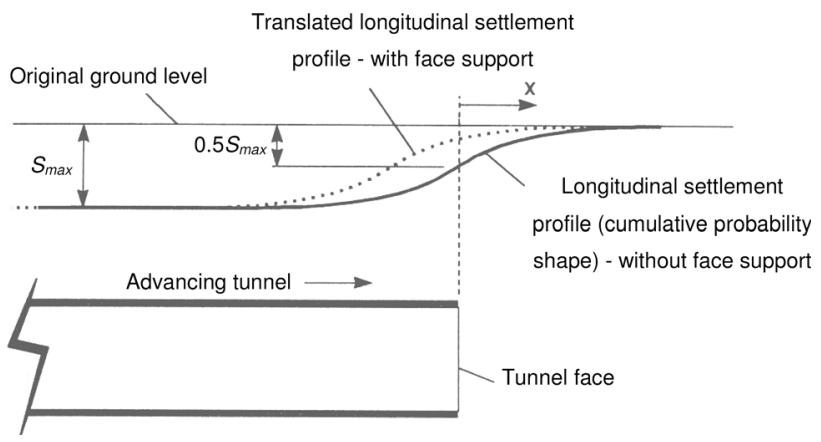

Fig. 12 Longitudinal Greenfield tunnel induced ground settlement (After Mair and Taylor [6])

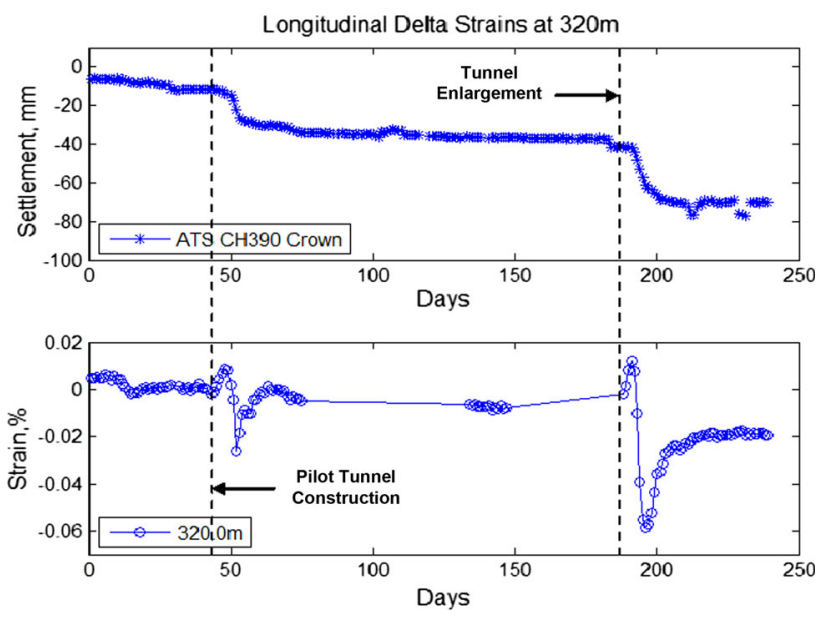

Fig. 13 Comparison of Automated Total Station (ATS) crown measurements and fibre optic strain (relative) data

over the same construction period. The vertical axis denotes the strain of the fibre optic cable (in percentage) where positive represents tensile strains. The longitudinal fibre optic cables were sufficiently sensitive to record small bending deformations during the pilot tunnel construction before picking up the larger strains induced by the full tunnel enlargement. In both construction stages, the hogging mode was observed initially (with tensile strain in the crown of the tunnel) before it rapidly changed into sagging mode where compressive strain was induced.

Measurements from the fibre optic system showed that the existing Royal Mail tunnel experienced bending mode deformations to some degree, where the linings rotated in relation to each ring towards the tunnelling face below, before levelling out again signified by the recovery of compressive strains. It should be noted from Fig. 13 that both systems recorded significant change in measurements at the exact same construction time period, signifying good agreement with the construction progress.

Interestingly, the longitudinal bending mode strains inferred from the fibre optics recovered almost completely for the pilot tunnel while approximately $30 \%$ of the 
residual compressive strains remained for the tunnel enlargement. The engineering implications of these residual strains require further investigation.

Back analyses were carried out for the pilot tunnel construction with the assumption of a linear strain distribution from the level of the L-brackets to the extreme fibre strains on the extrados of the cast iron lining. These strains were compared to the theoretical longitudinal Greenfield horizontal strains at the same level based on Attewell \& Woodman's equations [2] and presented in Fig. 14.

The extent of the settlement trough is controlled by the trough width parameter, K. At the ground surface, the average $\mathrm{K}$ of 0.5 for tunnels in clay was found to provide a reasonable fit from various field monitoring of tunnelling projects around the world. However, for subsurface settlement profiles, the trough width parameter increases with depth, given by Eq. 4.0 [5].

$K=\frac{0.175+0.325\left(1-z / z_{\mathrm{t}}\right)}{\left(1-z / z_{\mathrm{t}}\right)}$

Where $z$ is the depth from ground level to the level of interest while $z_{\mathrm{t}}$ denotes the depth to the new tunnel's axis from ground level. $\mathrm{K}$ was calculated to be 1.025 from the equation provided by Mair et al. [5] while the volume loss was assumed to be the designed value of $1.5 \%$; from other measurements on the same site the measured volume loss for the SCL tunnel construction was found to be reasonably close to $1.5 \%$.

The horizontal axis in Fig. 14 represents the normalised distance of the tunnel face from the point of monitoring. Negative $y / D$ indicates that the tunnel face lies ahead of the point of strain measurement, where $y$ denotes the

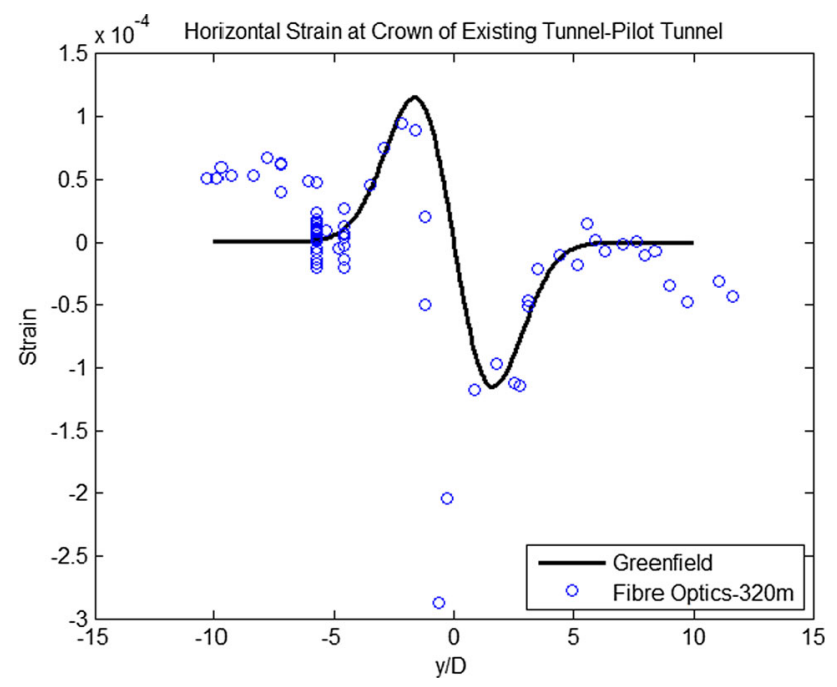

Fig. 14 Comparison of strains at lining extrados based on Greenfield and fibre optic measurements-Pilot tunnel construction longitudinal distance from the tunnel face to the measurement point and D is the diameter of the pilot tunnel.

General trends from both the theoretical and measured strains agree well with each other, despite the significantly higher material stiffness of the cast iron tunnel in comparison to the London Clay. This shows that the deformation of the Royal Mail tunnel was comparable to Greenfield settlements, a result with significant implications in practice.

Similar flexible response of existing tunnels was reported by Standing and Selman [13] in the Bakerloo and Northern lines during the construction of the Jubilee Line Extension (JLE) tunnels at Waterloo Station. JLE tunnels were $4.85 \mathrm{~m}$ in diameter and constructed approximately 6-11 m below the existing cast iron Bakerloo and Northern lines which were in London Clay. The existing running and station tunnels have a diameter of 3.6 and about $6.7 \mathrm{~m}$, respectively, and were aligned approximately in a perpendicular alignment, above the new JLE tunnels. Construction of JLE tunnels were carried out via open faced SCL tunnelling with $150 \mathrm{~mm}$ sprayed concrete lining reinforced with mesh and lattice girders.

Precise levelling measurements were taken from a point in the existing tunnel closest to the axis of JLE advancing face. It was found that these readings measured from the Bakerloo and Northern lines were in good agreement with the Greenfield cumulative probability function curves. This supports the notion that the structural stiffness of the existing tunnel would only have a minor influence on the settlement profiles; hence it will behave relatively flexibly.

Comparing the two idealised modes of deformation as discussed in "Sect. 2.1.2", deformation solely by the shear mode is less probable. Minimal restraints from the bolts will allow lining rings to rotate as well as settle vertically. Thus, a coupled partial shear and bending deformation mode is more conceivable.

The authors consider that this fibre optic installation to be only capable of measuring strains in the 'bending mode' of deformation (Fig. 2 upper); even though a small amount of geometry associated tensile strain will be imparted upon the longitudinal strain cable in the shearing mode (Fig. 2 lower); this second order movement is negligible. Therefore to explicitly measure shear displacement between two adjacent rings, wireless linear potentiometric displacement transducers (LPDT) were installed. LPDT sensors were also installed and aligned longitudinally to measure joint opening/closing, hence the bending mode.

LPDT sensors were mounted upon $3 \mathrm{~mm}$ steel plate 'sensor' brackets; themselves mounted to the circumferential flanges of the tunnel lining using M6 studs, preinstalled by drilling and tapping the cast iron flanges. The LPDT plungers acted upon a partnering 'target' bracket attached to the neighbouring tunnel ring flange. 
Compression of the LPDT sensor was read as negative, whereas extension, positive. The LPDT brackets were arranged so that compression of the shear LPDT would occur upon the tunnel ring closest to the advancing tunnelling face settling, whereas extension of the shear sensor would indicate heaving. The bending LPDT extends on joint opening, and compresses on joint closing, or hogging and sagging, respectively. The arrangement of LPDT sensors is shown in Fig. 15, with result data shown in Fig. 16.

Figure 16 shows movement recorded by both shearing and 'bending' LPDT sensors at a single location upon the tunnel crown in the vicinity of the fibre optic data presented in Fig. 13. Both pilot tunnel and later tunnel enlargement stages are shown with construction progress plotted bold solid and dotted against secondary (right) vertical axis, with bending and shearing data fine solid and dashed against primary vertical axis (left).

The LPDT sensors do not detect bending or shearing during the approach of the pilot tunnel. As the pilot tunnel progresses to be approximately in line with the sensors, both bending and shearing are detected simultaneously, recording $0.04 \mathrm{~mm}$ compression in bending and $0.06 \mathrm{~mm}$ in settlement shearing. As the pilot tunnel passes beyond the sensors, both shear and bending LPDT sensors indicate partial recovery, with $0.03 \mathrm{~mm}$ residual movement for both. It can be seen that the movements indicated are very small. To measure such small movement, the LPDT sensors used were low friction, contactless (resistive plastic) type, with effectively infinite resolution.
These were coupled to a 16 bit analogue to digital converter with amplifier.

During the later approach of tunnel enlargement, initial extension is recorded in the bending LPDT, indicating hogging, coincident with shear LPDT recording extension also, indicating heaving. Shortly ahead of the enlargement advance, bending and shear LPDT sensors have near returned to pre-tunnelling levels. As the tunnel enlargement advance now passes in line with the LPDT sensors, simultaneous compressive shearing and bending is recorded, with shearing peaking at $0.13 \mathrm{~mm}$ and bending peaking at $0.08 \mathrm{~mm}$. As the enlargement passes the sensors, a small recovery is detected, however residual shear is $0.05 \mathrm{~mm}$ greater than bending, with movements of $0.065 \mathrm{~mm}$ for bending and $0.115 \mathrm{~mm}$ for shear; seemingly now lockedinto the existing tunnel.

The shear and bending displacements were approximately $50 \%$ larger during the tunnel enlargement stage when compared to the construction of the earlier pilot tunnel. It is notable that the recorded compressive shear movements were approximately 6 and $43 \%$ greater than bending movements during these phases; indicating a compound bending and shearing mode of movement, with shearing component prevalent.

It is not straightforward to directly correlate LPDT data with that of fibre optics; although a hogging phase is evident during the enlargement stage, it was not detected during the pilot stage, indicating either the LPDT sensors are less sensitive than fibre optics, or the circumferential
Fig. 15 LPDT sensors and tunnel brackets mounting arrangement

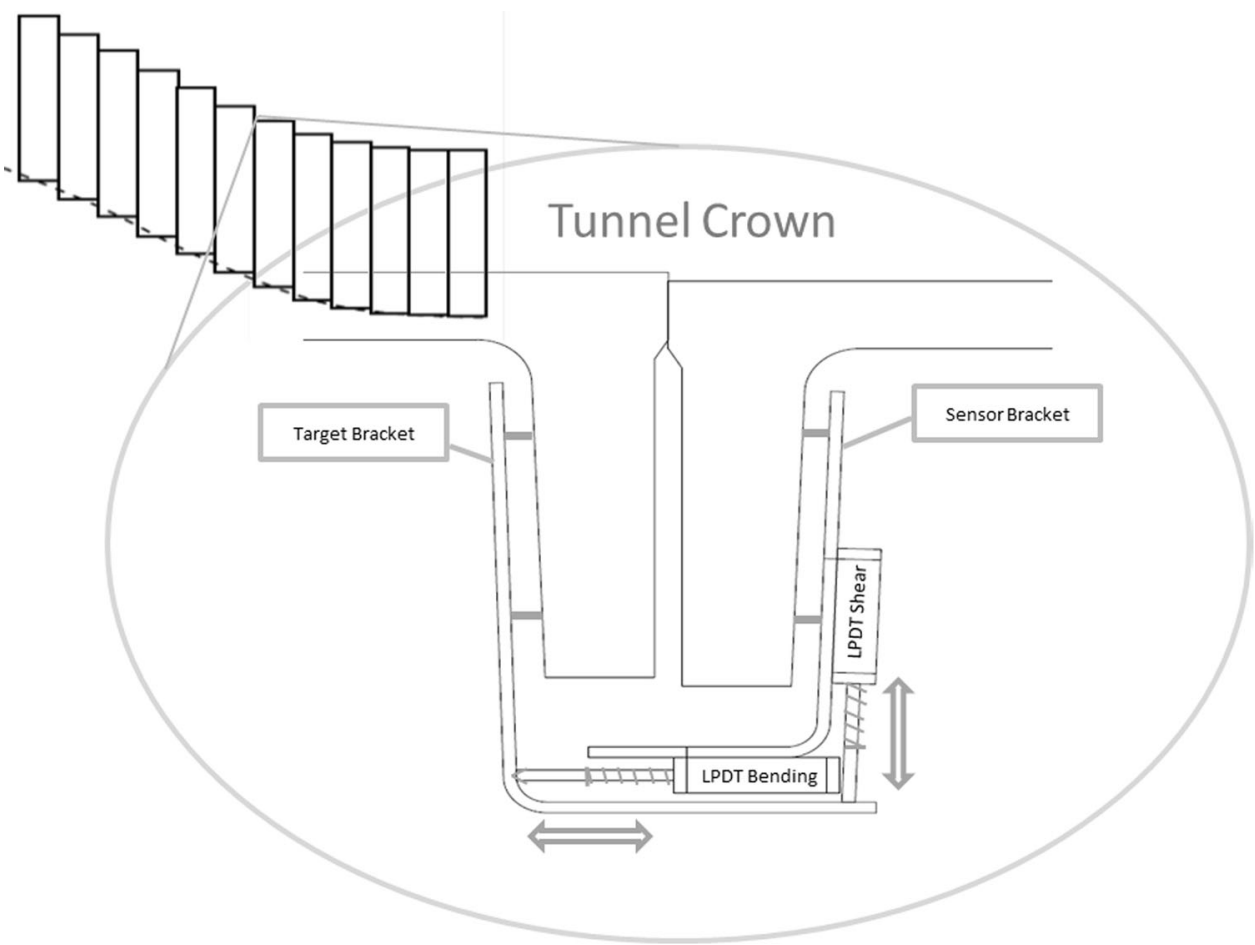


Fig. 16 LPDT bending and shearing response of a single circumferential connection due to pilot and enlargement stage tunnelling

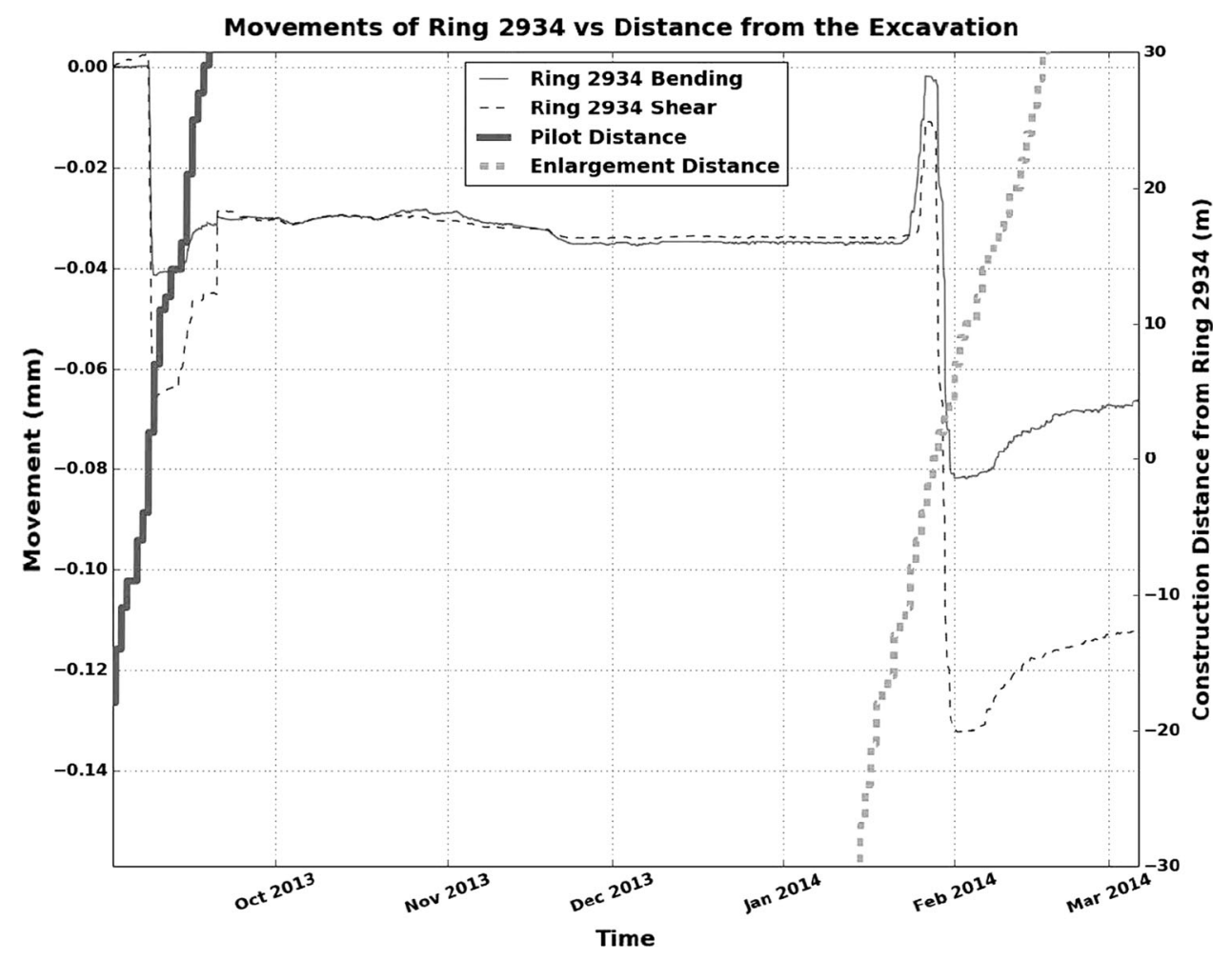

joints are sufficiently stiff to resist movement during this stage; the former is believed to be the more conceivable. At a local scale, we can observe that maximum movements occur at $y / D=0$ in agreement with fibre optic data.

A more useful consideration of LPDT data in this scenario is to correct fibre optic derived strain data by taking account of free movement at the joints as discussed in 3.2, effectively de-convoluting the fibre optic data. Considering a peak compressive strain of $\sim 0.05 \%$ was measured by the fibre optics and $0.07 \mathrm{~mm}$ detected by the bending LPDT at the same location, effective straining by free movement of the joint can be calculated by dividing LPDT movement by the tunnel ring width $(508 \mathrm{~mm})$ assuming that the cast iron material is completely rigid. Therefore $0.07 \mathrm{~mm}$ represents $0.014 \% \varepsilon$. On this basis, free movement of the joint accounts for $\sim 27 \%$ of the fibre optic derived straining; significantly reducing the material borne strain.

It is worth noting here that the shear LPDTs would not have been able to pick up any appreciable readings if the deformation was purely of the bending mode while the reverse is true for the fibre optic system. Thus, results from both fibre optic and wireless LPDT support the hypothesis of a compound movement of both bending and shearing displacements for a partial shear and bending mode. The results in Fig. 16 are not final as the LPDT sensors are undergoing further individual calibration to improve their accuracy. These early results are derived using bulk calibration factors. Hence a detailed review of this data is not conducted here.

\section{Conclusions}

Distributed fibre optic strain sensing with the BOTDR system has enabled a continuous strain profile of the existing cast iron Royal Mail tunnel, both in cross sectional and longitudinal direction, to be measured during the construction of Crossrail's platform tunnel less than $2 \mathrm{~m}$ directly below it. This has allowed a clear strain profile to be observed alongside the conventional displacement measurements of Automated Total Station for each tunnel construction stage.

Trends of longitudinal strains match very well with the crown settlement measured by the Automated Total Station system. The results indicate that the Royal Mail tunnel deforms flexibly, comparable to the cumulative probability curve of the longitudinal green field settlements. Much of the strains are recovered once the tunnelling face has advanced away from the measuring point.

In contrast to the longitudinal behaviour where the effects are short lived, the cross sectional strains are more permanent. Results from the cross sectional fibre optics has allowed a direct strain measurement to be obtained from the tunnel ovalisation. Relatively high strain readings were captured which was representative for the deformation of 
the entire ring as a whole. Further instrumentation such as LPDT sensors installed across the radial joints would be required to identify and separate the amount of strains that incur in the lining and its joints.

It must be stressed here that strains in the tunnel lining, rather than absolute displacements, are the governing parameter, which inform engineers on its structural health. Displacement measurements serve as a means to infer the strains that had developed within the lining indirectly. Limitation of target points of the conventional system of simply measuring displacements obscures the full mechanism and limits progress for future design improvements. This in turn encourages a conservative design approach to be adopted, potentially increasing the cost and schedule of construction.

Successful installation of a monitoring system extends beyond obtaining reliable measurements. Understanding the appropriate parameter to measure using appropriate instrumentation is imperative. Analogous to any instrumentation, distributed fibre optic strain sensing can only provide reliable measurements with satisfactory installations. It is hoped that this paper has provided some guidance on the essential considerations during the planning stage and on practical installation issues on site.

Further analyses and research currently continue at the University of Cambridge to study the behaviour of existing tunnels when subjected to tunnelling directly beneath it in close proximity.

Acknowledgments The authors would like to gratefully note the contributions in the form of logistical and technical support from Crossrail (particularly Mike Black, Stephen Roberts, Alison Norrish and Chris Dulake), Royal Mail (particularly Mitch Harris), ARUP (particularly Mike Devriendt), CH2 M Hill (particularly Peter Wright). This project would not have been possible without the financial support from Laing O'Rourke for the first author's PhD studentship as well as the continuous support from the UK Engineering and Physical Sciences Research Council (EPSRC) and Innovate UK through their funding of the Centre for Smart Infrastructure and Construction (CSIC) at Cambridge.

Open Access This article is distributed under the terms of the Creative Commons Attribution License which permits any use, distribution, and reproduction in any medium, provided the original author(s) and the source are credited.

\section{References}

1. Alhaddad M, Wilcock M, Gue CY, Bevan H, Stent S, Elshafie M, Soga K, Devriendt M, Wright P, Waterfall P (2014) Multi suite monitoring of an existing cast iron tunnel subjected to tunnelling induced ground movements. Geo-Shanghai International Conference 2014, Shanghai

2. Attewell PB, Woodman JP (1982) Predicting the dynamics of ground settlement and its derivatives caused by tunnelling in soil. Gr Eng 15(8):13-22

3. Duddeck H, Erdmann J (1982) Structural design models for tunnels. Proceedings Tunnelling 82, London, 83-91

4. Horiguchi T, Tateda M (1989) BOTDA- Nondestructive measurement of single-mode optical fibre attenuation characteristics using Brillouin interaction: theory. $\mathrm{J}$ Lightwave Technol 7(8):1170-1176

5. Mair RJ, Taylor RN, Bracegirdle A (1993) Subsurface settlement profiles above tunnels in clays. Géotechnique 43(2):315-320

6. Mair RJ, Taylor RN (1997) Theme lecture: bored tunnelling in the urban environment. In: Proceedings of the fourteenth international conference on soil mechanics and foundation engineering, Hamburg, 2353-2385

7. Mohamad H (2008) Distributed optical fibre strain sensing of geotechnical structures, PhD Thesis. Department of Engineering, University of Cambridge

8. Mohamad H, Bennett PJ, Soga K, Mair RJ, Bowers K (2010) Behaviour of an old masonry tunnel due to tunnelling-induced ground settlement. Géotechnique 60(12):927-938

9. Mohamad H, Soga K, Bennett PJ, Mair RJ, Lim CS (2012) Monitoring twin tunnel interactions using distributed optical fibre strain measurements. J Geotech Geoen Eng ASCE 138(8):957-967

10. Wood AMM (1975) The circular tunnel in elastic ground. Géotechnique 25(1):115-127

11. New BM, O'Reilly MP (1991) Tunnelling induced ground movements: predicting their magnitudes and effects. In: Proceedings 4th international conference on ground movements and structures, Cardiff. Invited review paper, Pentech Press, pp 671-697

12. Peck RB (1969) Deep excavations and tunnelling in soft ground. In: Proceedings of 7 th international conference of soil mechanics and foundation engineering, state of the art volume, pp 226-290

13. Standing JR, Selman R (2001) The response to tunnelling of existing tunnels at Waterloo and Westminster. Building response to tunnelling, Chapter 29, Vol. 2 Case studies from construction of the Jubilee Line Extension, London. CIRIA Special Publication vol 200. pp 509-546

14. Verruijt A, Brooker JR (1996) Surface settlement due to deformation of a tunnel in an elastic half-plane. Géotechnique 46(4):753-756 